\title{
CHROMOSOMAL INSTABILITY ASSOCIATED WITH DUPLICATIONS IN MAIZE
}

\author{
G. GAVAZZI, M. SANDRI, G. ANZANI and A. GHIDONI \\ Institute of Genetics, University of Milan, Milan, Italy
}

Received 4.xi.76

\begin{abstract}
SuMMARY
Strains of maize are described with a duplicate segment in a displaced position on the long arm of chromosome $10(\mathrm{Dp} 10-1,-2,-3,-4)$. The existence of the duplication is inferred from the linkage data, the decrease of recombination values in the region proximal to the duplicate segment and the drastic effect on growth and sex influorescence development observed in $\mathrm{Dp} 10$ homozygous plants.

Furthermore the analysis of the Dp 10-1 strand suggests that the presence of the extrachromatin elicits a chromosomal instability conducive to partial or complete loss of the duplicated segment.

Comparisons of quantitative values of the anthocyanins conditioned by a gene, $P$, either in its normal position or on the displaced duplication disclose significant differences in the pigment content values, suggesting that position effect phenomena might account for such differences.
\end{abstract}

\section{InTRODUCTION}

THE results presented in a previous paper (Gavazzi, 1975) dealing with the transmission and linkage values of an abnormal chromosome 10 in maize, suggested that this chromosome carries a duplicate region displaced to a new position. Strains homozygous for the displaced duplication have a characteristic morphology consisting of narrow leaves and poorly developed male and female inflorescences.

The morphogenetic effect is seemingly associated with the distal portion of the duplication. Furthermore, the analysis of the transmission behaviour of the presumptive duplicate strands indicated that such strands undergo frequent breakage-fusion-bridge (BFB) cycles in the endosperm tissues, suggesting that the presence of extra chromatin is conducive to chromosomal instability.

The present paper is concerned with such findings and extends them to other duplicate strands in order to clarify the following points:

(a) cytological evidence of the existence of a displaced duplication;

(b) chromosomal instability of the strand carrying additional chromatin, i.e. tendency of the duplicate strand to undergo partial or complete loss of the duplicate segment;

(c) comparison of the expression of a gene component of the $R$ locus affecting plant pigmentation $(P)$ in its standard position and in the dislocated duplication.

\section{Material AND methods}

(i) List of markers used

$R$-st $(p S-s t)$ : stippled aleurone, green plant;

$R$-sk $(p S$-sk): smoky aleurone, green plant; 
$R$-sc $(p S$-sc): self-coloured aleurone and scutellum, green plant;

$R$-pal 1 ( $p S$-pal 1): weakly pigmented aleurone and scutellum, green plant. Originally isolated as a somatic mutational sector on a homozygous $R$-sc plant, following ethyl-methane-sulphonate treatment of the seeds;

$R-r(P S)$ : coloured aleurone, red plant;

$r-r(P s)$ : colourless aleurone, red plant;

$R-g(p S)$ : coloured aleurone, green plant;

$r-g(p s)$ : colourless aleurone, green plant;

$R$-ch Stadler: coloured aleurone, anthocyanin developed in specific tissues of the plant. Specificity of pigment production in these tissues seemingly associated with different $R$ components (Sastry, 1970);

$g$ : golden plumule and dark yellow silks;

$M$-st: a stippled modifier that affects specifically the expression of $R$-st by enhancing the number of dark spots determined by $R$-st. Active both in cis and trans. Its loss leads to a light stippled phenotype;

$K$ : a cytological marker, consisting of a large heterochromatic segment attached to the end of the long arm of an abnormal chromosome 10 (K 10).

Symbols in brackets refer to the dual structure of the $R$ locus. The lower-case letters $p$ and $s$, as used in this context, do not distinguish between presence of a recessive allele or absence of the gene component.

All the markers used have the genetic background of the inbred line W 22 . The order of the genes and their map distances are given below:

$$
g 14 R 5.7 M-s t>36 K
$$

$R-r$, known as the standard allele, is a chromosomal region lying at position 57 on the long arm of chromosome 10, associated with two adjacent duplications of the type known as tandem repeats.

The genetic length of the duplicated segment amounts to 0.16 map units (Dooner and Kermicle, 1971).

In $R-r$ the proximal and distal members of the duplicated region are marked $P$ and $S$ respectively, $P$ conditioning plant, and $S$ seed pigmentation (anthocyanin). The structural organisation of the stippled allele $(R-s t)$ is less clearly defined (see Brink, 1973, for a review).

Occasionally derivatives of stippled are obtained with a different pattern of aleurone variegation or with self-coloured aleurone and scutellum.

The event leading to the occurrence of germinally transmissible selfcoloured kernels is mainly confined to meiosis or gametophyte development while that accounting for the pattern alleles takes place both at meiosis and in somatic cells.

The stippled derivative used in this work $(R-s k)$ is the result of a somatic event.

\section{(ii) Duplication strains}

The presumptive duplications here analysed were originally isolated in test-crosses of $R$-st/r-r $K 10$ or $R-s k / r-r K 10$ with $r-g r-g$ as non-parental seedlings carrying properties of both parental markers, i.e. stippled (or smoky) aleurone and red plant tissues.

Further studies proved they carry a non-parental strand carrying $P$ linked 
to the stippled factor. One of the isolates proved upon further testing to carry a duplicated region marked with $P$, distal to $R$ (Gavazzi, 1975).

In this work strands with a presumptive dislocated duplication are indicated by the symbol $\mathrm{Dp} 10$ and an isolation number.

Dp 10-1 was isolated from heterozygous $R-s t / r-r K 10$ females and Dp $10-2,-3,-4$ from $R-s k / r-r K 10$ males crossed with $r-g / r-g$ testers. The exceptional strands were recovered with a frequency of about $2.7 \times 10^{-4}$.

In previous reports (Gavazzi, 1975; Gavazzi and Anzani, 1976) Dp $10-1$ and 2 were referred to as case 1 and 2 respectively.

The symbol $\mathrm{N} 10$ applies to a chromosome 10 of normal constitution. When reference is made to the $P$ component of $R$ lying in the dislocated duplication the symbol $P_{\mathrm{Dp}}$ is used to distinguish it from $P$ in its standard position.

\section{(iii) Cytological preparations}

A few male inflorescences of plants carrying the duplication DP $10-1$ in the heterozygous condition were collected for the study of meiotic stages.

After fixation in a $3: 1$ alcohol-acetic mixture, the specimens were stored in a freezer. Acetocarmine smear preparations were made by the usual procedures for maize microsporocytes (McClintock, 1930).

\section{(iv) Quantitative anthocyanin determination}

Seeds were surface sterilised in a 5 per cent calcium hypochlorite solution for $15 \mathrm{~min}$., rinsed twice with distilled water and left in Petri dishes on wet filter paper in growth chambers $\left(t=22^{\circ} \mathrm{C}\right.$; continuous light of about 300 foot candles).

Three days after germination anthocyanins of primary roots were extracted by grinding root segments $5 \mathrm{~cm}$ long in a mortar with liquid nitrogen, adding a fixed volume of $0 \cdot 1$ per cent $\mathrm{HCl}$ ethanolic $(\mathrm{v} / \mathrm{v})$ solution. The extract was then centrifuged twice and the supernatants used for spectrophotometric reading at $530 \mathrm{~nm}$.

Each reading refers to a sample of 5 root segments of equal length.

\section{Results}

\section{(i) Linkage and transmission of the duplication strands}

As already mentioned under MATERIALs the four presumptive duplications represent independent events. They were originally isolated from $p S$-var $P$ s $K$ heterozygotes (where $S$-var stands for $S$-st or $S$-sk) as nonparental strands carrying $P$ in coupling with $S$-var. Evidence that these strands carry $P$ dislocated to a new position on the same chromosome is offered by the linkage data in test-crosses of $p S$-var $P_{\mathrm{Dp}} / p s$ with homozygous p $s$ males (table 1 ).

The $S-P_{\mathrm{Dp}}$ recombination values of the four duplication strains are not the same, suggesting that these duplications do not occupy the same position or, alternatively, that they involve segments of different length. Furthermore, the yield of the two complementary classes of non-parentals in Dp $10-1$ and 4 is not balanced. This difference, however, is accounted for by the low transmission of $\mathrm{Dp} 10$ gametes (see table 2) in the case of $\mathrm{Dp} 10-1$ while it appears real for $\mathrm{Dp} 10-4$. 
TABLE 1

Frequency of nonparental strands recovered in the progeny of heterozygous $\mathrm{p}$ S-var $\mathrm{P}_{D p} / \mathrm{p}$ semales crossed with $\mathrm{p} s$ males

\begin{tabular}{|c|c|c|c|c|c|c|}
\hline \multirow[t]{2}{*}{ Duplication } & \multicolumn{2}{|c|}{$S$-var } & \multirow[t]{2}{*}{$\begin{array}{c}\% \\
\text { non-par }\end{array}$} & \multicolumn{2}{|c|}{$s$} & \multirow[t]{2}{*}{$\begin{array}{c}\% \\
\text { non-par }\end{array}$} \\
\hline & $P$ & $p$ & & $P$ & $p$ & \\
\hline Dp 10-1 & 3623 & 116 & $3 \cdot 1 \pm 0.3^{*}$ & 56 & 3445 & $1.6 \pm 0.2 \dagger$ \\
\hline Dp-10-2 & 2181 & 95 & $4 \cdot 1 \pm 0 \cdot 4$ & 97 & 2299 & $4 \cdot 0 \pm 0.4$ \\
\hline Dp 10-3 & 844 & 37 & $4.2 \pm 0.7$ & 63 & 1146 & $5 \cdot 2 \pm 0.6$ \\
\hline Dp 10-4 & 308 & 7 & $2.2 \pm 0.8$ & 1 & 364 & $0.3 \pm 0.3$ \\
\hline
\end{tabular}

* and $\dagger$ Corrected values, on the basis of the weak competitive ability of Dp 10-1 carrying gametes (see table 2), are $2 \cdot 3 \pm 0 \cdot 2$ and $1 \cdot 9 \pm 0 \cdot 2$ respectively.

TABLE 2

Male and female transmission of the $D p 10$ strands as determined in test-crosses of $D p 10 / \mathcal{N} 10$ females with $\mathcal{N} 10 / \mathcal{N} 10$ males

$\begin{array}{lrccc}\text { Duplication } & n^{*} & \begin{array}{c}\text { Dp 10 female } \\ \text { transmission } \\ (\%)\end{array} & n^{*} & \begin{array}{c}\text { Dp 10 male } \\ \text { transmission }\end{array} \\ \text { Dp 10-1 } & 13241 & 42 \cdot 7 \pm 0 \cdot 4 & 3712 & 17 \cdot 2 \pm 0 \cdot 6 \\ \text { Dp 10-2 } & 6466 & 50 \cdot 3 \pm 0 \cdot 6 & 2498 & 55 \cdot 0 \pm 1 \cdot 0 \\ \text { Dp 10-3 } & 3583 & 49 \cdot 6 \pm 0 \cdot 8 & 1360 & 48 \cdot 7 \pm 1 \cdot 3 \\ \text { Dp 10-4 } & 3074 & 48 \cdot 9 \pm 0 \cdot 9 & 7474 & 22 \cdot 8 \pm 0.5\end{array}$

* Total no. of seeds scored.

The association of the dislocated $P$ with a duplicated segment distal to $R$ was previously inferred, in the case of $\mathrm{Dp} 10-2$, from the results of a threepoints linkage test as well as from the observation of a significant decrease in recombination in the regions adjacent to $R$ in $\mathrm{N} 10 / \mathrm{Dp} 10$ heterozygotes and the abnormal morphology of plants homozygous for the dislocated $P$.

We tentatively assume that the other three strands carry a duplicate segment on the basis of the observation that they share features exhibited by Dp $10-2$, such as the abnormal morphology and the decrease in recombination in the $R$ region (unpublished results).

Subsequently we shall validate this assumption for $\mathrm{Dp} 10-1$. The transmission of the duplicated strands (table 2) in the female germ line, as determined in test-crosses of Dp $10 / \mathrm{N} 10$ females, is normal except for Dp 10-1 which shows a transmission value slightly lower than that of a $\mathrm{N} 10$ strand. As to male transmission, the results indicate that $\mathrm{Dp} 10-1$ and four pollen grains have a weak competitive ability compared to N 10 pollen grains, while $\mathrm{Dp} 10-2$ and three grains have a normal transmission.

(ii) Cyclic and noncyclic breaks of $D p 10$ strands

Some of the variegated (stippled or smoky) kernels borne on test-crossed ears (see table 1) show somatic sectors of colourless tissues including $1 / 2-1 / 4$ of the endosperm surface or a pigment distribution typical of BFB cycles, suggesting that the $\mathrm{Dp} 10$ strand has undergone losses of chromatin, including or excluding the $R$ locus, during the endosperm divisions. 


\section{TABLE 3}

Frequency $\left(\times 10^{-3}\right)$ of breaks of $D p 10$ strands estimated on the basis of the number of smoky or stippled kernels with BFB cycles or large colourless sectors observed on test-crosses of $\mathrm{p} \mathrm{S-var} \mathrm{P}_{D p} / \mathrm{p} \mathrm{s}$ heterozygotes

\begin{tabular}{|c|c|c|}
\hline \multirow[t]{2}{*}{ Duplication } & \multicolumn{2}{|c|}{ Dp 10 entering the cross as } \\
\hline & 우우 & वे \\
\hline Dp 10-1 & $16 \cdot 8 \pm 1 \cdot 7$ & $25 \cdot 1 \pm 6 \cdot 2$ \\
\hline Dp $10-2$ & - & $2.9 \pm 1.4$ \\
\hline Dp 10-3 & $6 \cdot 7 \pm 1.9$ & $12 \cdot 1 \pm 4 \cdot 2$ \\
\hline Dp $10-4$ & $13 \cdot 3 \pm 2 \cdot 9$ & $76 \cdot 9 \pm 6 \cdot 4$ \\
\hline
\end{tabular}

The frequency of these events, given in table 3 is much higher when the duplication strand present in the endosperm is of paternal rather than maternal origin. This discrepancy is accounted for by the triploid constitution of the endosperm tissue, while the differences between the four duplication strains are possibly related to the nature of the duplications (position and extent).

\section{(iii) Lack of association between loss of the transposed duplication and recombination}

The high yield of kernels with a duplication strand undergoing BFB cycles and $R$ losses during endosperm development suggests that the presence of extra chromatin on chromosome 10 leads to chromosomal instability and to loss of the duplication.

If duplication strands undergo similar losses of the $P$ duplication during meiosis, then we would expect to observe an uneven yield of the two classes of non-parental in the progeny of $\mathrm{Dp} 10 / \mathrm{N} 10$ plants, $p S$-var $P_{\mathrm{Dp}} / p s$ in genotype, with an excess of $p S$-var over $p s P_{\mathrm{Dp}}$ strands. To test this possibility we made use of heterozygous $\mathrm{N} 10 / \mathrm{Dp} 10$ plants carrying the same Dp $10-2$ strand, marked $p S$-sk $P_{\mathrm{Dp}}$, but differing in the $R$ constitution of the $\mathrm{N} 10$ strand.

\section{TABLe 4}

Frequency of non-parental strands determined in test-crosses of $\mathcal{N} 10 / D p 10-2$ fermales differing in their $\mathrm{R}$ constitution

\begin{tabular}{|c|c|c|c|c|c|c|c|}
\hline \multirow{3}{*}{$\begin{array}{l}\text { Pistillate parent } \\
\text { genotype }\end{array}$} & \multicolumn{5}{|c|}{ Strand constitution of test-crosses: } & & \\
\hline & \multicolumn{2}{|c|}{ N 10} & \multicolumn{2}{|c|}{$\mathrm{Dp} 10$} & \multirow[b]{2}{*}{$n$} & \multicolumn{2}{|c|}{$\%$ Non-parental } \\
\hline & $p$ & $P$ & $p$ & $P$ & & N 10 & Dp 10 \\
\hline$R-s k / p S-s c P_{\mathrm{Dp}}$ & 645 & 33 & 91 & 473 & 1242 & $4 \cdot 87$ & $16 \cdot 13$ \\
\hline$R-s c / p S-s k P_{\mathrm{Dp}}$ & 1458 & 100 & 269 & 1368 & 3195 & $6 \cdot 42$ & $16 \cdot 43$ \\
\hline$R$-pal $1 / p S$-sk $P_{\mathrm{Dp}} *$ & 2764 & 177 & 308 & 2645 & 5903 & $6 \cdot 02$ & $9 \cdot 06$ \\
\hline$R-g / p S_{-s k} P_{\mathrm{Dp}}$ & 2604 & 88 & 225 & 2557 & 5474 & $2 \cdot 21$ & $7 \cdot 91$ \\
\hline$r-g / p S-s k P_{\mathrm{Dp}}$ & 1508 & 71 & 82 & 1256 & 2917 & $4 \cdot 49$ & $6 \cdot 01$ \\
\hline$R-r / p S-s k P_{\mathrm{D} p}$ & $-f$ & $-\dagger$ & 144 & 1720 & 1864 & - & $7 \cdot 72$ \\
\hline$R-c h / p S-s k P_{\mathrm{Dp}}$ & $-\dagger$ & $-\dagger$ & 203 & 2660 & 2863 & - & 6.67 \\
\hline
\end{tabular}

* In this and succeeding genotypes the frequencies of non-parentals are the values obtained on the basis of the progeny test results.

$\dagger$ The yield of non-parental N 10's is not detectable in this genotype. 


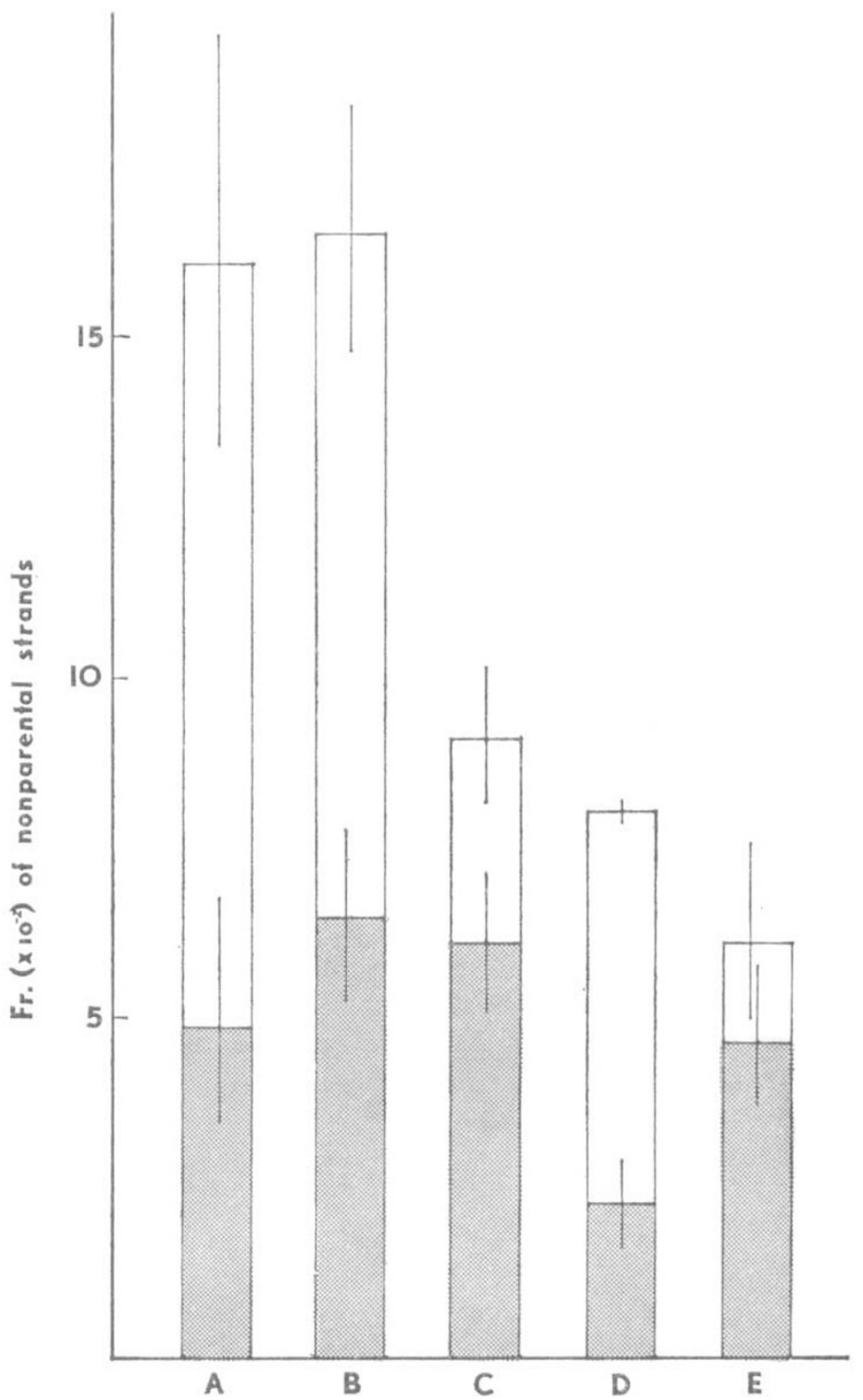

FiG. 1.-Frequency of non-parental strands recovered in test-crosses of Dp 10-2/N 10 females differing in their $R$ constitution with $\mathrm{N} 10 / \mathrm{N} 10$ males.

$$
\begin{aligned}
& \mathrm{A}=\frac{p S-s k}{p S-s c P_{\mathrm{Dp}}} ; \quad \mathrm{B}=\frac{p S-s c}{p S-s k P_{\mathrm{Dp}}} ; \quad \mathrm{C}=\frac{p S-p a l 1}{p S-s k P_{\mathrm{Dp}}} ; \quad \mathrm{D}=\frac{p S}{p S-s k P_{\mathrm{Dp}}} ; \\
& \mathrm{E}=\frac{p s}{p S-s k P_{\mathrm{Dp}}}
\end{aligned}
$$

(Don-parental Dp 10 strands; Non-parental N 10 strands; $\square$ Non-parental Dp 10 strands not accountable for by recombination). 
The heterozygotes were test-crossed and their progeny classified, after germination, for their $\mathrm{P}$ marker constitution. As anticipated, the data (table 4 and fig. 1) show that in each heterozygous Dp 10/N 10 arrangement allowing the recovery of both classes of non-parentals, the yield of nonparental Dp 10 ( $p S$-sk) exceeds that of the reciprocal non-parentals carrying $P$. The rate of loss of $P$ from the duplication strand ranges between 6 and 9 per cent except for the $p S$-sk $P_{\mathrm{Dp}} / p S$-sc heterozygote where the loss exceeds 16 per cent. This higher value is by no means related to the presence of $R$-sc on $\mathrm{N} 10$ since the same value is obtained in $p S$-sc $P_{\mathrm{Dp}} / p S$-sk parents.

\section{(iv) Linkage data in $D p 10-1$ strand}

Case 1 strand carries $M$-st distal to $S$-st. Its loss through crossing over gives rise to a light stippled phenotype.

If this strand carries $P$ distal to $R$, then the light stippled recombinants yielded by test-crosses of $p S$-st $M$-st $P_{\mathrm{Dp}} / p s+$ females should not carry $P$ but they still would if it were proximal to $R$. Out of 18 presumed light stippled progeny tested, nine bred true. Eight of them lost $P$, while one retained it. This is the result expected if the gene sequence of the strand is $S$-st- $M$-st-P. The number of putative light stippled originally isolated were 26 out 5658. Their frequency, estimated on the basis of the result of their progeny test, amount to 0.23 per cent, a much lower value than the expected $5 \cdot 7$ per cent.

As to the three-point test-cross data of $G S$-st $P_{\mathrm{Dp}} / g p s$ females, the following distribution of the $g$ marker was observed:

\begin{tabular}{|c|c|c|c|c|c|c|c|c|}
\hline \multirow[b]{2}{*}{$S-s t P_{\mathrm{Dp}}$} & \multicolumn{3}{|c|}{ Parentals } & \multicolumn{4}{|c|}{ Recombinants } & \multirow[b]{2}{*}{$n$} \\
\hline & & & & & & & & \\
\hline$G$ & $g$ & $G$ & $g$ & $G$ & $g$ & $G$ & $g$ & \\
\hline 2374 & 5 & 105 & 3210 & 62 & 9 & 49 & 0 & 5814 \\
\hline
\end{tabular}

These results are quite unexpected. Among parentals, in fact, $g$ recombinants are almost null in $S$-st $P$ and 3.16 per cent only in $p s$ strands, while among recombinants the frequency of $S$-st and $s$ strands carrying $g$ is about 23 and 0 per cent respectively (the $g-R$ recombination of normal strains in the W 22 genetic background amounts to about 19 per cent). The data suggest that the $\mathrm{Dp} 10-1$ strand carries in its long arm a duplicate region, 20 or more map units long, including the $G$ and $P$ markers.

The fact that all the $p s P_{\mathrm{Dp}}$ recombinants (49) carry the $G$ marker suggests that the transposed duplication is in reversed order.

\section{(v) Evidence for the presence of a long duplicated region in $D p 10-1$ strand}

Evidence in favour of the presence of the duplicated region was sought among the light stippled recombinants isolated in test-crosses of $G p S$-st $M$-st $P_{\mathrm{Dp}} / g p s+$ females. If the duplicated segment of Dp $10-1$ strand is distal to $R$ then the light stippled recombinants should have a normal strand without the transposed duplication and they would thus show normal values of recombination in the $G-R$ region. The results reported in table 5 confirm this expectation. 
TABLE 5

Tield of strands with non-parental $\mathrm{g}$ marker constitution as determined in test-crosses of plants obtained from presumptive light stippled kernels originally isolated on ears produced by $\mathrm{G} p \mathrm{p}$-st $\mathrm{M}-\mathrm{st} \mathrm{P}_{\mathrm{Dp}} / \mathrm{g} \mathrm{p}$ $\mathrm{s}+$ females crossed with $\mathrm{g} \mathrm{p} \mathrm{s}$ males

Female coded no.

1. Light stippled

$\begin{array}{lrr}2482 & 26 \cdot 1 & 3 \cdot 1 \\ 2648 & 25 \cdot 7 & 2 \cdot 2 \\ 2651 & 23 \cdot 6 & 3 \cdot 4 \\ 2484-1 & 22 \cdot 8 & 2 \cdot 5 \\ 2484-4 & 15 \cdot 3 & 2 \cdot 9 \\ 2484-6 & 19 \cdot 2 & 3 \cdot 2 \\ 2484-12 & 31 \cdot 7 & 2 \cdot 6 \\ 2486 \dagger & 4 \cdot 4 & 1 \cdot 0\end{array}$

2. Stippled

$\begin{array}{lll}2219 & 3 \cdot 5 & 0 \cdot 4 \\ 2220 & 2 \cdot 4 & 0 \cdot 3 \\ 2219-10 & 1 \cdot 6 & 1 \cdot 1 \\ 2219-11 & 2 \cdot 8 & 1 \cdot 6 \\ 2219-13 & 0 \cdot 8 & 0 \cdot 7 \\ 2484-15 & 5 \cdot 0 & 2 \cdot 2\end{array}$

Inferred Dp 10-1 strand constitution
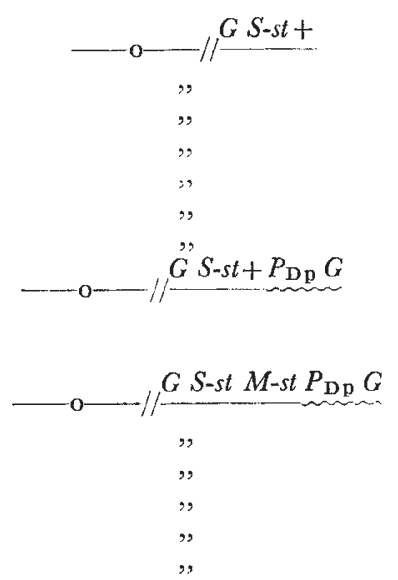

* Frequency of strands with non-parental $g$ marker constitution.

$\dagger$ Originally isolated as a somatic light stippled sector.

Further data in favour of the hypothesis come from the analysis of a sample of plants derived from stippled kernels with BFB cycle in their endosperm. These kernels, derived from test-crosses of $G p S$-st $P_{\mathrm{D} p} / g p s$ plants (see table 3 ) proved upon germination to have lost $P_{\mathrm{Dp}}$ in 8 out of 16 cases. Four of them were used in test-crosses to $g p s$ plants to see whether loss of $P_{\mathrm{D} p}$ is associated with re-establishment of normal transmission and $g-R$ recombination values. The results, reported in table 6 , indicate that only one of the four derivative strands lost a major portion or all of the

\section{Table 6}

Frequency of strands with non-parental $\mathrm{g}$ marker distribution as determined in the progeny of $D p 10-1 / \mathcal{N} 10$ heterozygotes reciprocally crossed with $\mathcal{N} 10$ homozygotes. The $D p 10-1$ strands and derivatives were originally recovered in a sample of stippled kernels with $B F B$ endosperm, yielded by test-crosses of G p S-st M-st $P_{\mathrm{Dp}} / \mathrm{g} \mathrm{p} \mathrm{s}+$ females with $\mathrm{g} \mathrm{p} \mathrm{s}+$ males

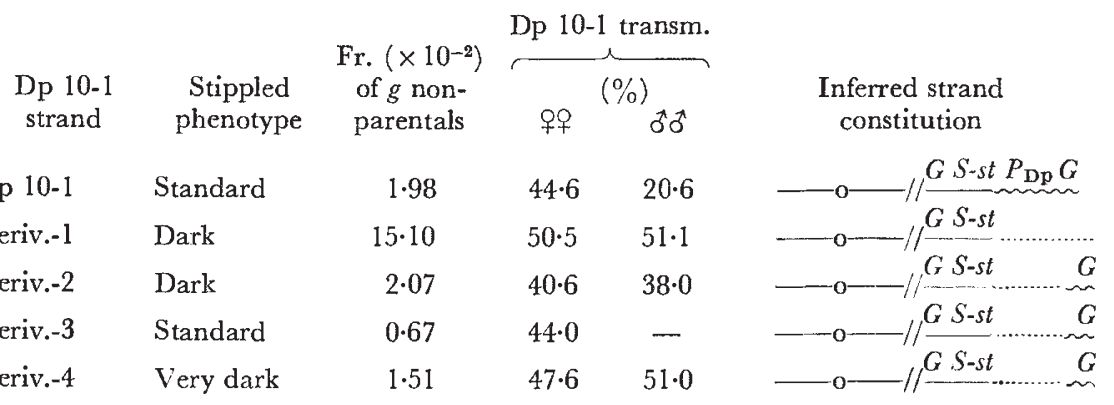


translocated duplication while the remaining three retained a portion marked with $G$. The apparent association of loss of the duplication or part of it with an altered stippled expression still remains unexplained.

\section{(vi) Cytological observations}

Plate 2 shows two typical pachytene configurations of heteromorphic chromosome 10 bivalents, when the duplication DP $10-1$ is present in the heterozygous condition.

In these pictures the extra segment in the duplication chromosome appears to amount to or exceed 50 per cent of the long arm.

Both the general aspect and the details of these pictures, together with genetic data (suggesting that both $P$ and the $g$ loci are present in the duplicated segment) are consistent with the presence of a duplication including a large portion of the long arm of chromosome 10 .

The other duplications $\mathrm{Dp} 10-2, \mathrm{Dp} 10-3$ and $\mathrm{Dp} 10-4$ were not cytologically analysed. In the present material the extra segment is often folded back over itself in various ways, to accomplish the irregular pairing one would expect.

\section{TABLE 7}

Anthocyanin content, expressed as A 530, of seedling primary roots in presence of one dose of $\mathrm{P}$ either in its normal position or on the displaced duplication

\begin{tabular}{|c|c|c|c|c|}
\hline Duplication & Root genotype & $n$ & $\begin{array}{c}\text { Anthocyanin } \\
\text { content } \\
\text { (A 530) }\end{array}$ & $\begin{array}{c}\% \\
\text { control }\end{array}$ \\
\hline N 10 & $p S-s t / P s$ & 20 & 0.571 & $100 \cdot 0$ \\
\hline Dp 10-1 & $p S$-st $P_{\mathrm{Dp}} / p s$ & 20 & 0.055 & $9 \cdot 6$ \\
\hline Dp $10-2$ & $p S$-sk $P_{\mathrm{D} \mathrm{p} / p s}$ & 20 & $0 \cdot 103$ & $17 \cdot 5$ \\
\hline Dp $10-2$ & $p s P_{\mathrm{D} \mathrm{p} / p} S-s k$ & 20 & $0 \cdot 100$ & $18 \cdot 0$ \\
\hline Dp 10-3 & $p S-s k P_{\mathrm{D} p} / p s$ & 20 & 0.067 & $11 \cdot 7$ \\
\hline Dp $10-4$ & $p S-s k P_{\mathrm{Dp} / p} s$ & 15 & $0 \cdot 071$ & $12 \cdot 4$ \\
\hline
\end{tabular}

\section{(vii) Effect of the displacement of the $\mathrm{P}$ bearing duplication on the anthocyanin content of sporophytic tissues}

In table 7 the root pigment concentration of $P s / p S$-var seedlings is compared to that of $p S$-var $P_{\mathrm{Dp}} / p s$ seedlings. The results indicate that one dose of $P_{\mathrm{Dp}}$, carried on the displaced duplication, conditions a significantly lower pigment content of sporophytic tissues than a $P$ gene in its normal position. The average pigment value of each of the four duplication strains amounts to between 10 and 20 per cent of that observed in the control strain, with $P$ in its normal position. Since the duplication strains carry $P_{\mathrm{Dp}}$ in coupling with $S$-var, the observed decrease in pigment might be accounted for by a cis effect of the stippling factor over $P$ gene action. This possibility, however, is made unlikely by the observation that the pigment value of $p s P_{\mathrm{Dp}} / p S$-var roots is still as low as that of the other duplication strains.

\section{Discussion}

Both the genetic and cytological data presented confirm the presence of a displaced duplication in the abnormal chromosome 10 analysed. Cytological data (Plate I) referring to $\mathrm{Dp} 10-1$, are best explained by the presence 
of a reverse tandem duplication in the long arm of chromosome 10. The duplicated chromosome is possibly lacking the distal portion of the normal chromosome 10. In favour of this interpretation is also the fact that such duplications, when homozygous, yield off-type plants with narrow leaves and poorly developed inflorescences.

The genetic analysis of the duplication strands discloses a chromosomal instability leading to partial (see Dp 10-1) or complete loss of the duplicate segment. New strands can thus be derived carrying regions duplicated and deleted to different extents. Losses of the dislocated duplications appear to occur during endosperm development (see table 3), possibly as a consequence of meiotic events, as well as in the germ cells (see table I). In the latter the loss rate of the duplication is seemingly related to the degree of structural homology at the $R$ locus, as the data of table 6 suggest.

An unexpected feature disclosed by this study is the observation of a seemingly differential gene activity of $P$ according to its chromosomal location. Root pigment content of $p S$-var $P_{\mathrm{Dp}} / p s$ seedlings in fact amounts to only 10-20 per cent of that of $p S$-var/P $s$ seedlings; $P_{\mathrm{Dp}}$ of Dp I0 $-\mathrm{I},-3$ and -4 exhibiting the highest reduction ( 10 per cent of standard $P$ ) and $P_{\mathrm{Dp}}$ of Dp 10-2 yielding about 20 per cent of the expected pigment content. Since we do not have information on the relationship between $P$ and $P_{\mathbf{D p}}$, the possibility exists that the differences in pigment content reflect allelic differences of the two $P$. Against this possibility stands the fact that the duplication strains differ in the extent of reduction in pigment potential. It seems more likely that, since $P_{\mathrm{D} p}$ was originally isolated from $R$-var $/ r-r K$ parents, the transfer of its genetic information from one chromosome to its homologue took place in a way that affected the structure of $P$. In this case the decrease of anthocyanin associated with $P_{\mathbf{D} p}$ would be related to a structural change at $P$. On the other hand the lower pigmentation conditioned by $P_{\mathrm{D} p}$ could be accounted for by a position effect.

If the $R$ gene cluster works as a functional unit, one would expect the disruption of the unit to affect the gene action of the components of the system, leading to a partial impairment of their activity. It might also be that the low pigment values are an effect of the chromosomal imbalance as such.

Acknowledgments.-This research was partially financed by C.N.R. (Italian National Council Research, Rome).

The technical assistance of $\mathrm{Mr} \mathrm{G}$. Todesco is gratefully acknowledged.

\section{REFERENCES}

BRINK, R. A. 1973. Paramutation. Ann. Rev. Genet., 7, 129-153.

DOONER, H. K., AND KERMICLE, J. L. 1971. Structure of the $R-r$ tandern duplication in maize. Genetics, 67, 427-436.

GAVAZzI, G. 1975. Transposition of a member of a duplicated region in maize. Heredity, 35, 389-400.

GAVAZZI, G., AND ANZANI, G. 1976. A second case of transposed duplication in chromosome 10. Maize Genet. Coop. News Letter, 50, 74-77.

MC CLINTOCK, B. 1930. A cytological demonstration of the location of an interchange between the nonhomologous chromosomes in Zea mays. Proc. Nat. Acad. Sci., U.S.A., 16, 791-796.

SAStry, G. R. K. 1970. Paramutation and mutation of $R$-ch in maize. Theoret. Appl. Genet., 40, 185-190. 

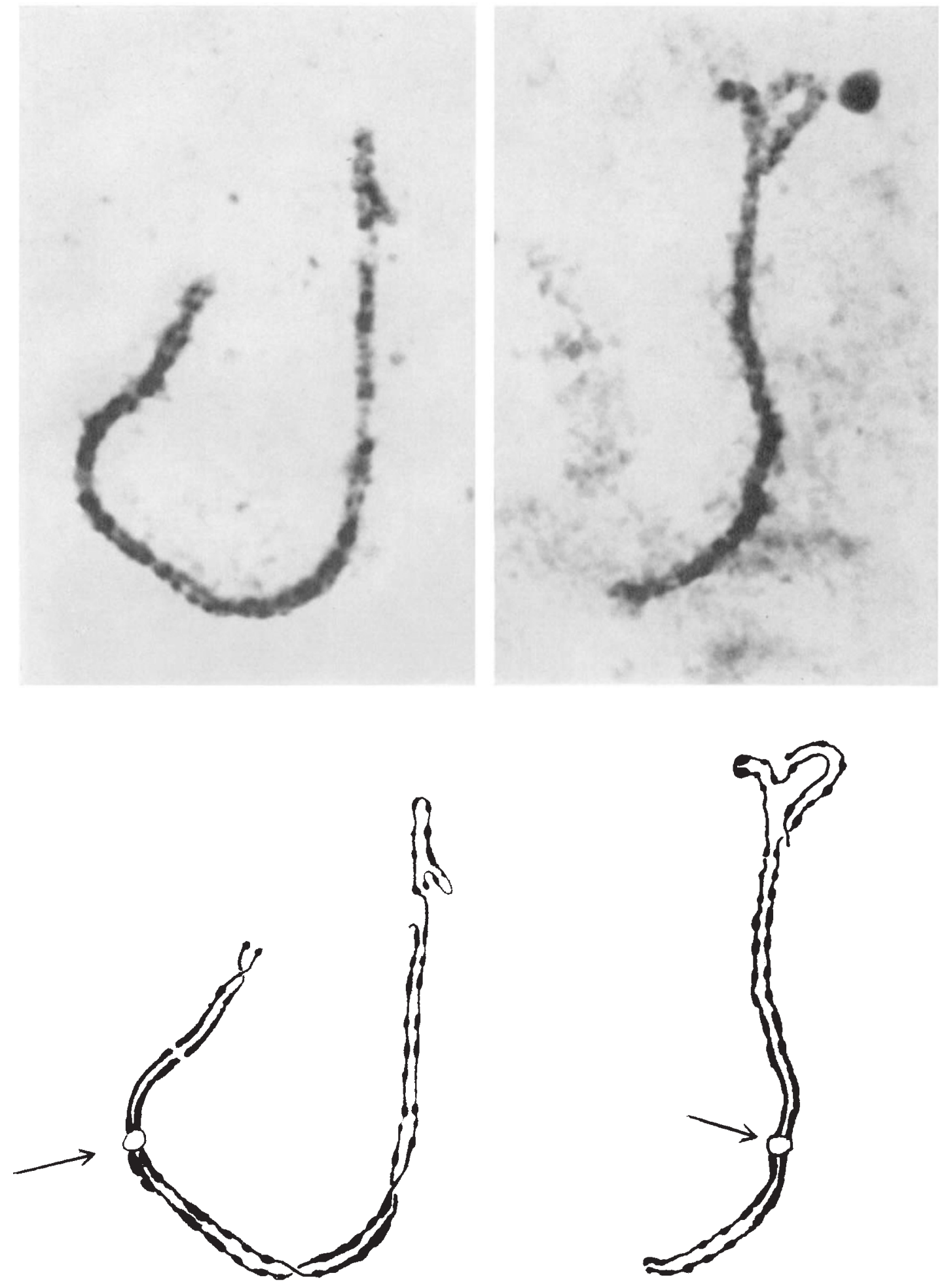

Pl.ATE I.--Two pachytene configurations ( $a$ and b) observed with heteromorphic bivalents involving a normal chromosome 10 and a chromosome 10 carrying the duplication Dp $10-1$ in the long arm. The interpretation is given in the camera lucida drawings ( $a^{\prime}$ and $\left.\mathrm{b}^{\prime}\right)$. Magnification: $\times 1,500$ (arrows point centromere position). 